\title{
Tailoring super-hydrophobic properties of electrochemical biosensor for early cancer detection
}

\author{
Natalia Malara ${ }^{1,5, *}$, Francesco Gentile ${ }^{2}$, Lorenzo Ferrara ${ }^{3}$, Marco Villani ${ }^{4}$, Salvatore Iannotta ${ }^{4}$,
} Andrea Zappettini ${ }^{4}$, Enzo Di Fabrizio ${ }^{5,6}$, Valentina Trunzo ${ }^{1}$, Vincenzo Mollace ${ }^{1}$, Nicola Coppedé ${ }^{4}$

\author{
${ }^{1}$ Department of Health Science, University of Magna Graecia, 88100 Catanzaro, Italy \\ ${ }^{2}$ Department of Electrical Engineering and Information Technology, University Federico II, Naples, Italy \\ ${ }^{3}$ Istituto Italiano di Tecnologia, Via Morego 30, 16163 Genova, Italy \\ ${ }^{4}$ IMEM-CNR Parco Area delle Scienze 37/A - 43124 Parma, Italy \\ ${ }^{5}$ Department of Experimental and Clinical Medicine, University of Magna Graecia, 88100 Catanzaro, Italy \\ ${ }^{6}$ King Abdullah University of Science and Technology, Thuwal, Saudi Arabia
}

* corresponding author: nataliamalara@unicz.it

\begin{abstract}
In this paper, we demonstrate an organic electrochemical transistor (OECT) based on the conductive polymer PEDOT:PSS for the analysis of the cell culture medium upon interaction with circulating cells isolated form peripheral blood sampling of health, sub-clinical and cancer patients. The device comprises arrays of super-hydrophobic micro-pillars in which a finite number of pillars incorporates nano-electrodes for site specific measurements of a solution. Due to its nano-scale architecture, the device realizes time and space resolved measurement of biological solution. Tumor metabolism could produce reactive species able to determine a different electronic behavior of correspondent microenviroment. On this basis, the device here presented the changes in the ESR signals was used to identify electronic changes occurring in the analysis of different type of microenvironment. Our results demonstrate that the device is able to register significative difference to differentiate healthy individuals form cancer patients, through an easy blood sampling. In conclusion, these preliminary data are suggestive of a novel test potentially useful to early identification of subjects at risk to development cancer disease.
\end{abstract}

\section{INTRODUCTION}

Conducting polymers are materials displaying high electrical conductivity, easy of fabrication, flexibility and biocompatibility, for this, they are routinely employed in organic electronics, printed electronics, and bioelectronics [1-3]. Organic electrochemical transistors (OECTs) are a second generation of Organic Thin Transistors in which a conductive polymer interacts directly with the electro-active medium, that is, the electrolyte. OECT devices based on the conductive polymer PEDOT:PSS have been demonstrated in chemical and biological sensing: while accurate in determining the size of individual ions in solution $[4,5]$, similar devices break down if challenged with complex mixtures, due to the lack of spatial resolution. Here, we modified a conductive PEDOT:PSS polymer to include extra non-continuous scales in the device (Figure 1). We fabricated arrays of superhydrophobic SU8 micro-pillars (Figure 1a) in a non-periodic square lattice, in which the distance between the pillars smoothly transitions from the center to the periphery of the pattern [6] (Figure 1b). The pattern, in turn, incorporates a finite number of nano-electrodes in a line that represent the sensitive spots of the device (Figure 1c-d). The entire system is coated with a conductive PEDOT:PSS polymer and by a fluorocarbon polymer to assure hydrophobicity [7] (Fifgure 1d). A solution on a similar device would maintain a spherical shape as suspended in air (Figure 1e). Due to its curvature, 
Marangoni convective flows develop within the volume of a drop of solution[8-10]. The competition between convection and diffusion will cause a spatial separation of biological species that would depend on the size and charge of the species in a solution. The incorporation of nano-scale details permits site specific current measurements of a sample.
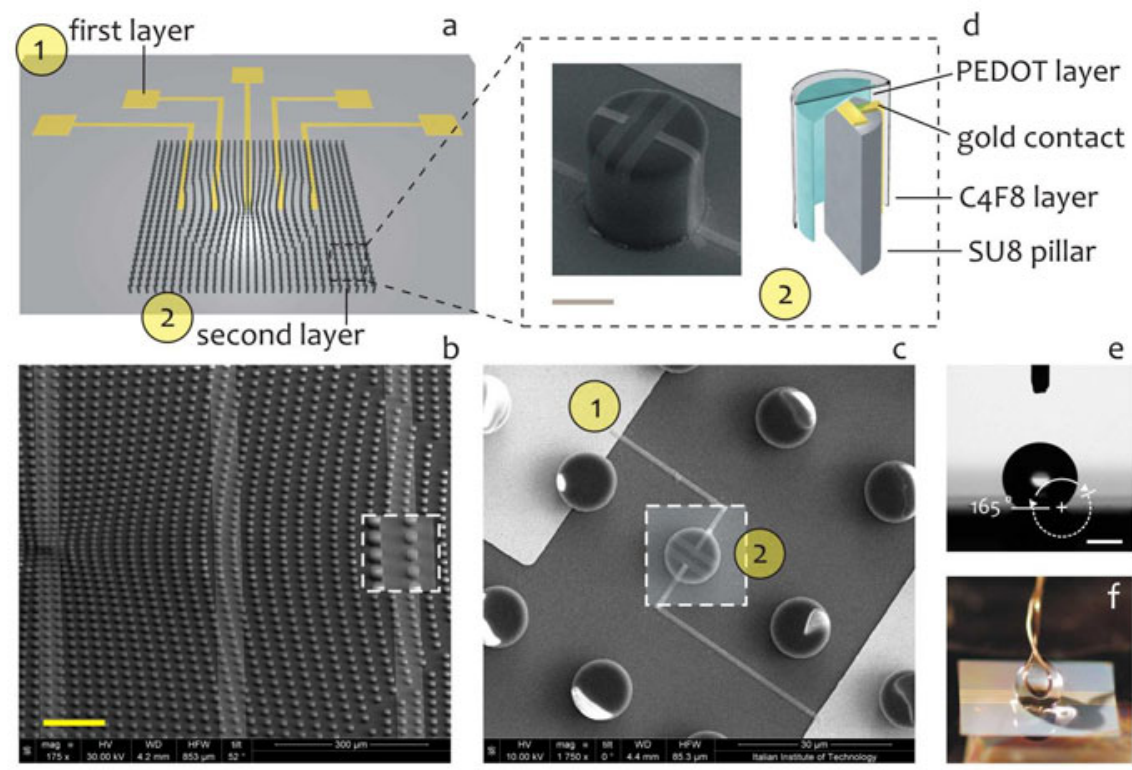

Figure 1 Schematic of the device, this comprises super-hydrophobic pillars aligned on conducting gold contacts (a). SEM micrograph of the pillars, the scale bar corresponds to $100 \mu \mathrm{m}$ (b). Some pillars of the device incorporates nano-scale contacts for site specific measurements of the device, the scale bar corresponds to $10 \mu \mathrm{m}(\mathrm{c}, \mathrm{d})$. The contact angle of solutions positioned on the device is as large as $165^{\circ}$, the scale bar corresponds to $2 \mathrm{~mm}$ (e). Operating the device (f).

In the present paper, we operate the analysis of the cell culture medium upon interaction with circulating cell isolated form peripheral blood sampling of health, sub-clinical and cancer patients. Our group here propose an innovative point of view. Normally the human normal cells are resident in their referenced tissue immerged in interstitial liquid with an electrolytic state in turn dependent by the semi conductivity state of proteins. Perturbations of this electrolytic state are translate on perturbations on the semi conductivity state of proteins, structural proteins and relative insoluble and soluble fractions, with changes on electron spin resonance (ESR) signal. One of the defence mechanisms of electrolytic state of physiological microenvironment is the glyoxalase/methylglyoxal enzyme system. It was 1965 when Szent-Gyorgyi first stressed that ubiquitous glyoxalase/methylglyoxale enzyme system can be considered as responsible for the elimination of the aldehyde and as indirect regulator of semi conductivity state of proteins. In particular, methylglyoxal inhibits cell growth, while the glyoxalase system by breaking down methylglyoxal may act as a promoter of cell growth [11,12]. As a 
consequence of the theory, it is rational to postulate that a difference in glyoxalase activities should exist between neoplastic and normal tissue. On the other hand, the cancer cells are also characterized by a higher demand for increased rate of glycolysis. When the glycolysis is operative, the glyoxalase pathway is crucial for the early forms of glucose breakdown and remained preserved for detoxification of an $\alpha$-oxoaldehydes produced. The changes in the rate of glycolysis is the first perturbation reflecting changes in cell division or tumor proliferation. On these bases, we proposed the analysis of the liquid of cultivation of cells isolated by peripheral blood. The culture mediums were conditioned during a short-time cultivation of fourteen days performed as previously described [13]. This time is sufficient to enrich the culture medium of soluble fraction of proteins produced by cells $[14,15]$. Moreover, different metabolic condition and generation of free radicals can influence its electrolytic state. The changes in the ESR signals are analyzed with the devise here presented. The investigation was aimed to define an easy system to register the early electronic changes occurring in the microenvironment around the human cultivated cells to unmask precancerous state.

\section{EXPERIMENTAL DETAILS}

Fabrication of the devices. Super-hydrophobic substrates were fabricated. These are given by the superposition of different layers (Figure 1). Layer A is a silicon substrate with conductive gold circuits connecting the sensors in the inner part of the device to the source and drain electrodes positioned at the border of the device. Layer B comprises super-hydrophobic SU8 micro pillars in a non-periodic lattice. 5 pillars at the center of the motif were modified to incorporate metallic nanocontacts using EBID (Electron Beam-Induced Deposition) techniques [16]. The entire substrate is coated with a conductive PEDOT:PSS thin film. A fluorocarbon polymer $\left(\mathrm{C}_{4} \mathrm{~F}_{8}\right)$ is finally deposited on the devices [7].

Layer A. We used P-doped, (100) silicon wafers with 5-10 Ohm/cm resistivity as substrates. We spin coated a 1 um thick layer of S1813 (MicroChem) on the substrate @4000 rpm for $60 \mathrm{~s}$, then we baked the sample@9 $90^{\circ} \mathrm{C}$ for 4 minutes. We exposed the resist with optical lithography (Mask Aligner, MA6/BA6, SUSS MICROTEC) to obtain circuit patterns on the silicon substrate. We evaportated a $70 \mathrm{~nm}$ thick layer of gold on the sample; then we performed a lift-off process in acetone (AllChemicals) for 5 minutes to remove the un-exposed resist from the substrate and define the pattern of gold circuits.

Layer B. We spin coated the sample with a 15 um thick layer of SU8-25 (MicroChem) @3000 rpm for $60 \mathrm{~s}$, then we pre-baked it $@ 95^{\circ} \mathrm{C}$ for 25 minutes. We aligned a second optical lithography mask on the sample using conveniently positioned alignement markers. We exposed the sample with UV radiation to obtain a non-periodic lattice of SU8 pillars, in which the distance $\delta$ between the structures varies not linearly with the distance from the center of the lattice as in [7]. The variable tiling is responsible for a non-uniform surface energy distribution, which recalls the drop at the center of the device. The diameter $d$ and height $h$ of the pillars were adjusted as $d=10 \mu m$ and $h=20 \mu \mathrm{m}$. The masks necessary for optical lithography were written using direct laser writing (DWL66fs, HEIDELBERG Instruments).

In synthesis, the device is has been realized with a sequence of materials as follows: Silicon substrate, gold contacts, SU8 pillars, top contact in gold on 5 pillars, PEDOT:PSS and fluorocarbon polymer. 
SEM Characterization. SEM micrographs of the samples were acquired using a Dual Beam (SEMFIB) - FEI Nova 600 NanoLab system. In measuring the pattern of SU8 polymeric pillars, we used a $5 \mathrm{keV}$ energy and corresponding electron current of $0.98 \mathrm{pA}$.

Measuring the electrical activity of species in solution. Analytes in diluted solutions were positioned on the surfaces. The electrical activity of the device was measured using a 2-channel source/measure precision unit (Agilent B2902A) similarly to other reported experiments $[4,5]$. Upon application of a positive gate potential $5 \mathrm{gs}$, cations are transported from the electrolyte to the PEDOT:PSS channel [4] and a dedoping process is initiated. Biosensor current response is expressed as current modulation $\Delta \mathrm{I} / \mathrm{I}_{0}=\left(\mathrm{I}-\mathrm{I}_{\mathrm{o}}\right) / \mathrm{I}_{\mathrm{o}}$, where 5 is the drain current $5 \mathrm{ds}$ value measured for $5 \mathrm{gs}>0 \mathrm{~V}$ and $5_{\mathrm{o}}$ is the $5 \mathrm{ds}$ value for $5 \mathrm{gs}=\mathrm{o} \mathrm{V}$. The current traces have been acquired over at least 20 repetitions per gate potential, in which the repetitions have been realized in a short time interval and this guarantees that the experimental conditions are maintained constant (including the temperature and air humidity) over the whole duration of the experiment, that in turn assures reproducibility. Under these conditions, the amount of variation of values around the average is vanishingly small and thus negligible. Thus, the errors in measuring the currents have been calculated but are not represented in the diagrams because small respect to the average $(\mathrm{e}<0.01)$. Pair-wise comparisons between means of different groups were performed using a Student's 5 -test (two tailed, unpaired) where, for each couple of normally distributed populations, the null hypothesis that the means are equal was verified. Everywhere in the text the difference between two subsets of data is considered statistically significant if the Student's 5 -test gives a significant level 5 (5 value) less than 0.05 .

Collection of conditioned medium. Biological samples were extracted from healthy, subclinical and tumoral populations using the protocols described in [13]. All patients were enrolled at from February 2011 at University of Catanzaro and were informed of the investigational aspects of this study and provided written consent in accordance with local and international institutional guidelines. Local ethical committee approved the study with the number: 2013.34. We measured conditioned cell culture mediums extracted from tumoral, sub-clinical, and healthy patients enrolled following clinical/instrumental selection criteria detailed in the approved project. All peripheral blood samples collected amounted to $5 \mathrm{ml}$ of volume. Ficoll-Paque Plus was used for cancer cells separation and the $1.080-1.090 \mathrm{~g} / \mathrm{ml}$ phase was identified as working density phase [15]. The cellular suspension isolated in correspondence of the working density phase was recovered in specific medium. The cell culture was expanded for 14 days (short-term cultivation) and the conditioned medium (aged 14 days old) was collected [14].

\section{RESULTS}

The device as in Figure 1 comprises arrays of super-hydrophobic micro-pillars that can manipulate biological solutions (Figure 1e). Positioned on the device, a biological sample maintains a quasispherical shape (Figure 1d, e) with a curvature at the border of the drop. This curvature generates convective fluxes that couple with diffusion to determine the trajectories of molecules in the volume of the drop [12]. In the device, 5 pillars, from border to center, are modified to incorporate microelectrodes (Figure 1c) for site specific measurements of the solutions. These permit to realize space and time resolved analysis of biological solutions. Using the described device, we measured conditioned cell culture mediums extracted from tumoral, sub-clinical, and healthy patients. To improve statistical analysis of the measurements, the current traces have been acquired over at least 20 repetitions per gate potential, in which the repetitions have been realized in a short time interval, this 
guarantees constant conditions as described in experimental part. Under these conditions, the amount of variation of values around the average is vanishingly small and thus negligible.
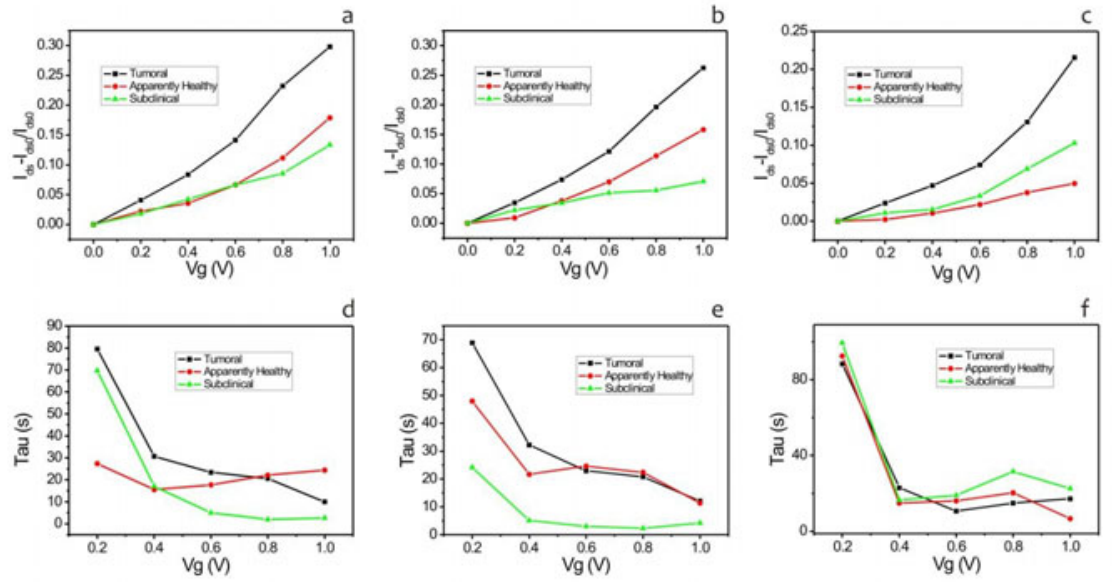

Figure 2 Ids current modulation measured on peripherally left (a), on the center (b), and peripherally right (c) of the device. The time constants reveal the kinetics of the analytes for the left (a), center (b), and right (c) transistors on the device.

Modulation amplitude of Ids in function of different gate voltages are reported in Figure 2. Ids current flows through Pedot:PSS film, between gold contacts on top of the pillars. This film area represents the channel of the transistor, the device gate is applied through the solute drop, by a Pt wire. In this configuration the cations and the positive charged molecules, as the gate voltage is applied, are forced to enter the PEDOT:PSS film, reducing its conductivity. The amplitude of this variation in the Ids current is proportional to the concentration of the cations, which in our case is related to the drop geometry and to the effects of the internal forces in the fluids. In Figure $\mathbf{2}$ the modulation, as expected, increase for higher gate voltages. Three different position are analysed for the different samples. Out of five different transistors, we report the results of three: the central one (Figure 2b), and the two external devices on both the left (Figure 2a) and right (Figure 2c) sides. Three culture medium have been analysed: (i) tumoral cells, (ii) healthy cells and (iii) sub-clinical cells, as described in the experimental section. In Figures 2a-c for all the considered positions on the device, the tumoral cells present a higher modulation amplitude, with a substantial difference with the other samples. The subclinical and the healthy samples present a lower amplitude of modulation and a less sharp increase. Between the Subclinical sample and the Healthy the differences are not substantial. In Figure 2a the two samples have the same amplitude, only at 0.8 and $1 \mathrm{~V}$ of potential the

healthy present a little increment. The same behaviour is present in the central device. While in the device on the opposite side, 0.8 and $1 \mathrm{~V}$ present a higher modulation for the subclinical sample. Characteristic time constants of the samples are reported In Figures 2d-f for different positions. The time constants tau represent the rising time of the device. These present a decreasing trend with the increase of gate voltages. At higher voltages some samples present constant or inverse trend. In 
particular, in Figure 2d and Figure 2e the tumoral sample present a higher characteristic time, while the subclinical and the

healthy results faster. In Figure $\mathbf{2 f}$ the samples present quite similar time constants, only with health for $1 \mathrm{~V}$ of gate.

\section{DISCUSSION}

We proposed the analysis of the liquid of cultivation of cells isolated by peripheral blood on the hypothesis that the conditioned medium can store modifications of the cellular metabolism. In fact, the tumor metabolism could produce reactive species able to determine a different electronic behavior of correspondent microenviroment. On this basis, the device here presented, has revealed the changes in the ESR signals analysing the different type of liquid of cultivation. The analysis of the results present interesting differences between the tumoral samples and the other cases. The most evident and clear difference is that the tumoral sample present a higher modulation, which increase faster with higher gate voltages, for all the samples and the device. Instead health and subclinical samples present lower modulation amplitude. The difference between the subclinical and healthy is not univoque, on one side and in the central position the healthy is higher but on the other side the subclinical invert the situation. Moreover from 0.2 to $0.6 \mathrm{~V}$ of gate voltage the two non tumoral samples are similar. Regarding the time constants on one side and in the central position, the tumoral sample present a higher time constants, especially respect to subclinical sample, while in the other side the constants are similar. Between the non tumoral samples subclinical results the faster in the modulation kinetic. The effect of the position is clearly showed in the figures, even if the central position present a difference, there is a tendency of an asymmetry respect to one side of the drop. These asymmetric difference influence the time constants as much as the modulation amplitude. Finally, the effect of a higher modulation in amplitude combined with a slower kinetic in the time constants for the tumoral samples, allows a significant distinction between tumoral and non tumoral samples. These characteristic distinction marks in the sensing process allows to apply the biosensing system as a promising, instruments to early cancer detection.

\section{CONCLUSIONS}

The primary human cell cultivation is a phase needed to test ex vivo the cellular metabolism. the Correspondent conditioned medium collected, with different composition in proteins and in reactive chemical species, is determinant to induce the different behaviour registered by the device. The good discrimination between tumor and non-tumor cases is the first point encouraging for further investigation on this way. However, the implementation of the number of cases is the first requisite to discriminate between healthy conditions by subject exposed to oxidants (environmental or drugs etc) by subject ongoing to neoplastic transformation. Further analysis to validate the conditioned medium as reference of the tumor microenviroment in vivo will be performed. Our final aim is to design a novel and easy approach for a real early cancer detection through a simple blood sampling.

\section{ACKNOWLEDGMENTS}


This work has been partially funded from the Italian Minister of Health (Project n. GR-2010-2320665) and by the project Interregional Research Centre for Food Safety \& health (IRC_FSH) (cod. PON a300359), granted to the Department of Health Science, University Magna Graecia. NC acknowledges Nicola Zambelli and Giacomo Benassi for technical support. NM acknowledges Prof. Bruno Silvestrini for scientific support. The authors would like to thank the patients and all health professionals involved at the University "Magna Graecia.

\section{REFERENCES}

[1] C. D. Dimitrakopoulos and P. R. L. Malenfant, "Organic Thin Film Transistors for Large Area Electronics," Advanced Materials, vol. 14, pp. 99-117, 2002.

[2] R. M. Owens and G. G. Malliaras, "Organic Electronics at the Interface with Biology," MRS Bulletin, vol. 35, pp. 449-456, 2010.

[3] G. Tarabella, C. Santato, S. Y. Yang, S. Iannotta, G. G. Malliaras, and F. Cicoira, "Effect of the gate electrode on the response of organic electrochemical transistors," Applied Physics Letters, vol. 97 , p. 123304, 2010.

[4] N. Coppedè, M. Villani, and F. Gentile, "Diffusion Driven Selectivity in Organic Electrochemical Transistors," Scientific Reports, vol. 4, pp. 1-7, 2014.

[5] F. Gentile, N. Coppedè, G. Tarabella, M. Villani, D. Calestani, P. Candeloro, et al., "Microtexturing of the Conductive PEDOT:PSS Polymer for Superhydrophobic Organic Electrochemical Transistors," BioMed Research International, pp. 1-10, 2014.

[6] F. Gentile, M. L. Coluccio, E. Rondanina, S. Santoriello, D. Di Mascolo, A. Accardo, et al., "Non periodic patterning of super-hydrophobic surfaces for the manipulation of few molecules," Microelectronic Engineering, vol. 111, pp. 272-276, 2013.

[7] F. Gentile, G. Das, M. Coluccio, F. Mecarini, A. Accardo, L. Tirinato, et al., "Ultra low concentrated molecular detection using super hydrophobic surface based biophotonic devices," Microelectronic Engineering, vol. 87 pp. 798-801, 2010.

[8] D. Tam, V. von Arnim, G. H. McKinley, and A. E. Hosoi, "Marangoni convection in droplets on superhydrophobic surfaces," Journal of Fluid Mechanics, vol. 624, pp. 101- 123, 2009.

[9] Y. Astier, L. Data, R. P. Carney, F. Stellacci, F. Gentile, and E. Di Fabrizio, "Artificial SurfaceModified Si3N4 Nanopores for Single Surface-Modified Gold Nanoparticle Scanning," Small, vol. 7, pp. 455-459, 2010.

[10] F. Gentile, M. L. Coluccio, R. P. Zaccaria, M. Francardi, G. Cojoc, G. Perozziello, et al., "Selective on site separation and detection of molecules in diluted solutions with superhydrophobic clusters of plasmonic nanoparticles," Nanoscale, vol. 6, pp. 8208-8225, 2014.

[11] M. P. Kalapos, "On the promine/retine theory of cell division: now and then," Biochimica et Biophysica Acta (BBA) - General Subjects; vol 1426, Issue 1, pp.1-16, 1999.

[12] D. Piskorska, R. Grabowska-Bochenek, "Role of glyoxalases and methylglyoxal in cell proliferation and differentiation," Postepy Hig Med Dosw, vol. 49, issue 3, pp. 433-44, 1995.

[13] N. Malara, M. Coluccio, T. Limongi, M. Asande, V. Trunzo, G. Cojoc, et al., "Folic acid functionalized surface highlights 5-methylcytosine-genomic content within circulating tumor cells," Small, vol. 10, pp. 4324-31, 2014.

[14] N. Malara, V. Trunzo, U. Foresta, N. Amodio N, S. De Vitis S, L. Roveda, et al., "Ex-vivo characterization of circulating colon cancer cells distinguished in stem and differentiated subset provides useful biomarker for personalized metastatic risk assessment," J Transl Med., vol. 14:133, pp. 1-12, 2016. 
[15] N. Malara, F. Givigliano, V. Trunzo, L. Macrina, C. Raso, N. Amodio, et al., "In vitro expansion of tumour cells derived from blood and tumour tissue is useful to redefine personalized treatment in non-small cell lung cancer patients," J Biol Regul Homeost Agents, vol. 28(4), pp. 717-31, 2014.

[16] F. Gentile, L. Ferrara, M. Villani, M. Bettelli, S. Iannotta, A. Zappettini, et al., "Geometrical Patterning of Super-Hydrophobic Biosensing Transistors Enables Space and Time Resolved Analysis of Biological Mixtures," Scientific Reports, vol. 6, pp. 1-15, 2016. 